\title{
Operation of Whale Optimization Algorithm in PID Controllers for AVR System
}

\author{
Sujatha Nebarthi I K M M Tarakesh
}

Department of Electrical and Electronics Engineering, N S Raju Institute of Technology, Sontyam, Visakhapatnam, 531173, A.P. India.

\section{To Cite this Article}

Sujatha Nebarthi and K M M Tarakesh, "Operation of Whale Optimization Algorithm in PID Controllers for AVR System", International Journal for Modern Trends in Science and Technology, 6(9): 01-05, 2020.

\section{Article Info}

Received on 06-August-2020, Revised on 20-August-2020, Accepted on 25-August-2020, Published on 28-August-2020.

\section{ABSTRACT}

In the present paper presents the Whale Optimization Algorithm technique (WOA) it is a partial search algorithm. To advance the improved the performance of the PID controller uses whale optimization algorithm as the optimization technique. The proposed algorithm is used to tuning the controllers very fast and tuning is very high quality in PID Controllers is most effectively. It growths the system by its main transient response and by comparing the all terms of rise time ( $\left.t_{r}\right)$, settling time $\left(t_{s}\right)$ and peak overshoot $\left(\% M_{p}\right)$. More over the three gains are (proportional $\left(k_{P}\right)$, integral $\left(k_{i}\right)$ and derivative $\left.\left(K_{d}\right)\right)$ of the PID controller have been enhanced by the WOA technique to control the Automatic Voltage Regulator system. In this the transient response of the terminal voltage may be observed from the well-conditioned analysis they can be suggest WOA established PID Controller and which reveal a very most upgrade strong control structure for the managing the AVR system in the Electrical Power System. The simulation result of the propounded controller has shown superior result to the other optimization techniques on PID controller along with the transient response parameters and improve and supervise the performance of the System.

KEYWORDS: PID controller, AVR - Automatic Voltage Regulator, WOA-Whale Optimization Algorithm

\section{INTRODUCTION}

In industries many of controllers are used but one of the highest utilized dominance is the proportional-integral-derivative (PID) controller which is implement for the improvement of the nature of steady state and along with the transient state [1]. The PID controllers are also named as in other term i.e. integer or conventional order controllers [2]. Another type of PID controller is there, which depends on the fragmentary value of the derivative and integral controller, called Frationary Order PID Controller (FOPID) [3]. Together with all the conventional PID controller parameters are (integral controller $-K_{i}$, proportional controller $-K_{p}$, and derivative controller $-K_{d}$ ), the FOPID has two extra parameters which are $m$ i.e. fractional value of derivative and 1 i.e. fractional value of integration.The system becomes more robust and it also more flexible when these two parameters are optimized, which are having fractional values, gets added with the PID controller [4].The production engineers are to be designing for the dissimilar approach are to be similar with the distinct value of the prearranged values and the actual values, which are accompanied from the System. Out of all these techniques, few are numerical and other 
techniques are to be attuned, partial or proceeding of the fuzzy control, etc. [5].

\section{AUTOMATIC VOLTAGE REGULATOR}

In nature, the most major element in the generation unit, even as the terminal of the alternator it neutralises the output voltage by regulating the excitation voltage of the generating system [6]. Undergoing the any kind of the weighted problem, the AVR system is design in such a way that would shall be monitoring continuing, for the

alternator's terminal voltage [7]. The AVR system contains of four familiar parts which are generator, amplifier, exciter, and sensor as represented in Fig. 1. Analytically, the functionalities of these elements in the AVR system they can be represented in a direct form instance the transfer function of sensor, exciter, amplifier and generator is appear in Table 1 being with the period of the gains and time constant of each element [8]. The other values of the element's gain and time constant, which is employed for the considered system, is also described in Table [9].

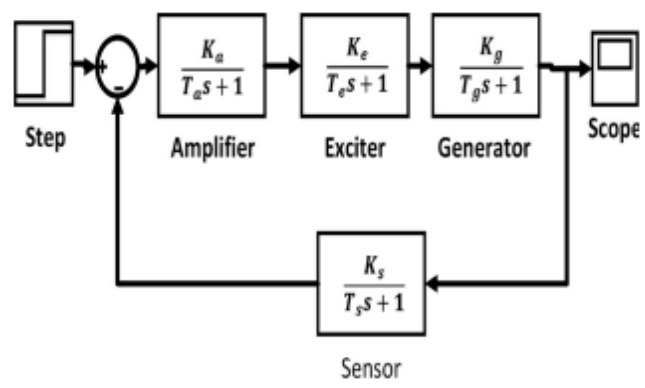

Fig. 1. Schematic diagram of the analysis of AVR system

\subsection{Representation of AVR System}

Representation of AVR system contains four elements in present four are the exciter, sensor, amplifier and generator. So these elements are create as four transfer function in which ignored are saturation and nonlinearity as shown in Fig. 3 using MATLAB/SIMULINK [10]. From which the major elements of the transfer function equations, their gains, ranges and time constants recycled are illustrated in Table $1[9,11]$.
TABLE I. TRANSFER FUNCTION AND STANDARDS OF THE AVR SYSTEM

\begin{tabular}{|l|l|l|l|}
\hline $\begin{array}{l}\text { AVR } \\
\text { component }\end{array}$ & $\begin{array}{l}\text { Transfer } \\
\text { Function }\end{array}$ & Range of $\mathrm{K}$ & $\begin{array}{l}\text { Range of } \\
\mathrm{T}(\mathrm{s})\end{array}$ \\
\hline Amplifier & $\mathrm{Ka} / 1+\mathrm{sTa}$ & $10 \leq \mathrm{Ka} \leq 40$ & $\begin{array}{l}0.02 \leq \mathrm{Ta} \\
\leq 0.1\end{array}$ \\
\hline Sensor & $\mathrm{Ks} / 1+\mathrm{sTs}$ & $0.9 \leq \mathrm{Ks} \leq 1.1$ & $\begin{array}{l}0.001 \leq \mathrm{Ts} \\
\leq 0.06\end{array}$ \\
\hline Generator & $\mathrm{Kg} / 1+\mathrm{sTag}$ & $0.7 \leq \mathrm{Kg} \leq 1$ & $\begin{array}{l}1.0 \leq \mathrm{Tg} \\
\leq 2.0\end{array}$ \\
\hline Exciter & $\mathrm{Ke} / 1+\mathrm{sTe}$ & $1 \leq \mathrm{Ke} \leq 10$ & $\begin{array}{l}0.4 \leq \mathrm{Te} \\
\leq 1.0\end{array}$ \\
\hline
\end{tabular}

The entire transfer function, consists of the transfer function of all this things, of the AVR system may be described in (1) and afterwards consideration of the charge of $\mathrm{Kg}$ and $\mathrm{Tg}$ as 1.0 and $1.0 \mathrm{~s}$, respectively.

$$
\begin{aligned}
& G_{A V R}(\mathrm{~s})=\frac{\Delta v_{t(s)}}{\Delta v_{\text {ref }}(s)} \\
& =\frac{0.5 s+10}{0.0004 s^{4}+0.045 s^{3}+0.444 s^{2}+1.61 s+12} \\
& =\frac{260(s+100)}{(s+98.92)(s+13.63)\left(s^{2}+2.057 s+22.05\right)}
\end{aligned}
$$

From (1), the characterized that only one zero and there be in the transfer function i.e. $Z=-100$. Forward with the one zero, two real poles are there at some poles are $S_{1}=-98.92$ and $_{2}=-13.63$ and $_{3,4}=-0.43 \pm \mathrm{j} 3.66$, two complex poles are also there. If zero and one pole is eliminated at 100 and -98.97 respectively, then the comparative value of the total transfer function, $G_{A V R}$ (s) and that inclined equation (2)

$G_{A V R}(\mathrm{~s})=\frac{250}{(s+13.63)\left(s^{2}+2.057 s+22.05\right)}$

Now, the AVR system the transfer function of the Amplifier, Exciter, Generator and Sensor of the $G_{A V R}(s)$ it may varies from zero and two real poles of the system. 


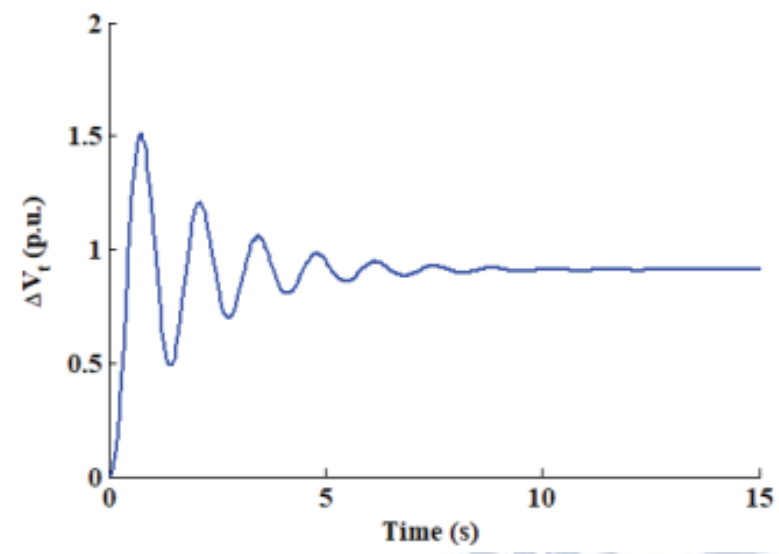

Fig. 2 AVR's terminal voltage output (outwardly controller)

In Fig. 2, the examined for AVR system the transient response is shown. From the diagram, it could be shown that the high fluctuation has been displayed by the transient response of the step parameters values.

$M_{p}$ as 1.5065 p.u, $T_{R}$ as $0.41 \mathrm{~s}, T_{S S}$ as $6.943 \mathrm{~s}$ and $E_{S S}$ as 0.0909. All things are reported from the figure with the terminal voltage $\Delta \mathrm{Vt}$ is contrast from these linear values. The deflection, which can be appearing in the transient response, is not adequate it also not desirable for the practical achievement of the alternator in the power generating system as the deflection is obtain in the unit of kilo-volt (KV). The performance of a robust controller is accompanied in the AVR system. The magnitudes of the guidelines which are being utilized for the representation of the AVR system are: $k_{a}=10.0, T_{a}=0.2 \mathrm{~s}, K_{e}=1.0, T_{e}=0.5 \mathrm{~s}, K_{e}=$ $2.0, T_{s}=0.02 \mathrm{~s}$. The load can decides the values of $K_{g}$ and $T_{g}$.

\section{PID CONTROLLER}

The proportional-integral-derivative controller is one of the simple structure, and all over is easy to construct and use, robust in nature, simple in structure, robust in nature and high degree of adaptability [12]. As the PID controller can be minimize the steady-state error, and the fastness of the responses can be increased by the controller and as well as the improvement of the transient response is also observed which makes the PID controller as a most of the chosen controller by the researchers [13]. These characteristics of the PID controller it encourage the researchers to accept the PID controller for their research purpose and this is the reason that the researchers have preferred the PID controller for the AGC problem also. The PID controller's model can be designed with the help of the transfer function, and in Laplace domain, it is presented in the where the parameters of controller shows the $K_{p}, K_{i}$ and $K_{d}$ which may stand for the gain of the proportional, integral and derivative individually. During the simulation, the magnitude of the $\mathrm{x}$ has been taken as 0.01 .

The PID controller is usually implemented as follows:

$$
\begin{array}{r}
\mathrm{u}(\mathrm{t})=K_{p}\left(e(t)+\frac{1}{T} \int_{0}^{t} e(t) d t+T_{d} \frac{d e(t)}{d t}\right) \\
\mathrm{e}(\mathrm{t})=y_{d}(t)-y(t) .
\end{array}
$$

Here, $\mathrm{u}(\mathrm{t})$ is the monitor output, $\mathrm{y}(\mathrm{t})$ and $y_{d}(t)$ are the process output and desired output of the system respectively. The object of the PID controller as to be study and is to be examine a set of gains are: $k_{p}, T_{i}$ and $T_{d}$, To improve the transient response of a system by compressing the overshoot and by reduction the settling time of the given system [14].

\section{Whale Optimization Algorithm}

WOA is newly formed metaheuristic optimization technique in which that might also can be seen that like in humans, whales are also having spindle cells due to which they have been their emotions, and they grow into judgemental and shows its action in the communal. Bubble net feeding is a specialized method which can be used by the humpback whale for getting into the prey by generating the bubbles after dip down in the rainwater. The Schema chart of the WOA is shown in Fig. 4 [7].

The following are the steps of WOA:

1) In the first initialized (agent is searched) the population number is designed along with all variables to the examined and maximum number of iterations are there in WOA.

2) For the particular search agent and the fitness function has to be calculated the best result i.e. By picked up for each optimum solution has to be fluctuate and update of the position to the best one also occurs.

3) For the particular search agent, the position can be updated by the following: a) If $R<0.5$, where $R$ is 
denoted as the random number which evasion in between 0 and 1 .

a) If $\mathrm{R}<0.5$, $\mathrm{R}$ represents random number

i) If $|\vec{X}|<1$ the situation that has to be renew by occupy the enclosing prey approach where:

$$
S(\vec{t}+1)=S *(t)-(\vec{X} * \vec{D})
$$

where the update position is $S(\overrightarrow{t+1})$ and the best result is $S *(t)$.

$\vec{X}=(2 * \vec{a} * \vec{r})-(\vec{a})$

where there is a decrease in the value from 2 to zero, in the value of $\vec{a}$ linearly and the random vector $\vec{r}$ evasion between $[0,1]$.

$\vec{D}=\mid(2 * \vec{r} * S *(t))-(\overrightarrow{S(t))} \mid$

Where, the position vector described as $\overrightarrow{S(t)}$.

ii) If $|\vec{X}|>1$, in the condition the position will be updated by utilize the phase method's exploration where:

$S(\vec{t}+1)=S^{\text {rand }}-(\vec{X} * \vec{D})$

$\vec{D}=\mid\left(2 * \vec{r} * \overrightarrow{S^{\text {rand }}}\right)-(\overrightarrow{S(t))} \mid$

where $\overrightarrow{S^{r a n d}}$ is the random position vector.

b) If the value of $\mathrm{P}>0.5$, By implementing the spiral form the position will be change. where :

$$
S(\overrightarrow{t+1})=\left(\overrightarrow{D^{\prime}} * e^{b l} * \cos (2 \pi L)\right)+S *(t)
$$

where the random number $\mathrm{L}$ and spiral shaped logarithmic is stand by $b$ evasion between -1 and 1 and

$\overrightarrow{D^{\prime}}=S * \overrightarrow{(t)}-\overrightarrow{S(t)}$

4) The 2nd step and the 3rd step has to be replicated for after the renew of the position while the fitness function has to be calculated and to selected the best position will be chosen until the iteration ability its maximum value and hence the selection of the optimum solution is go through in system.

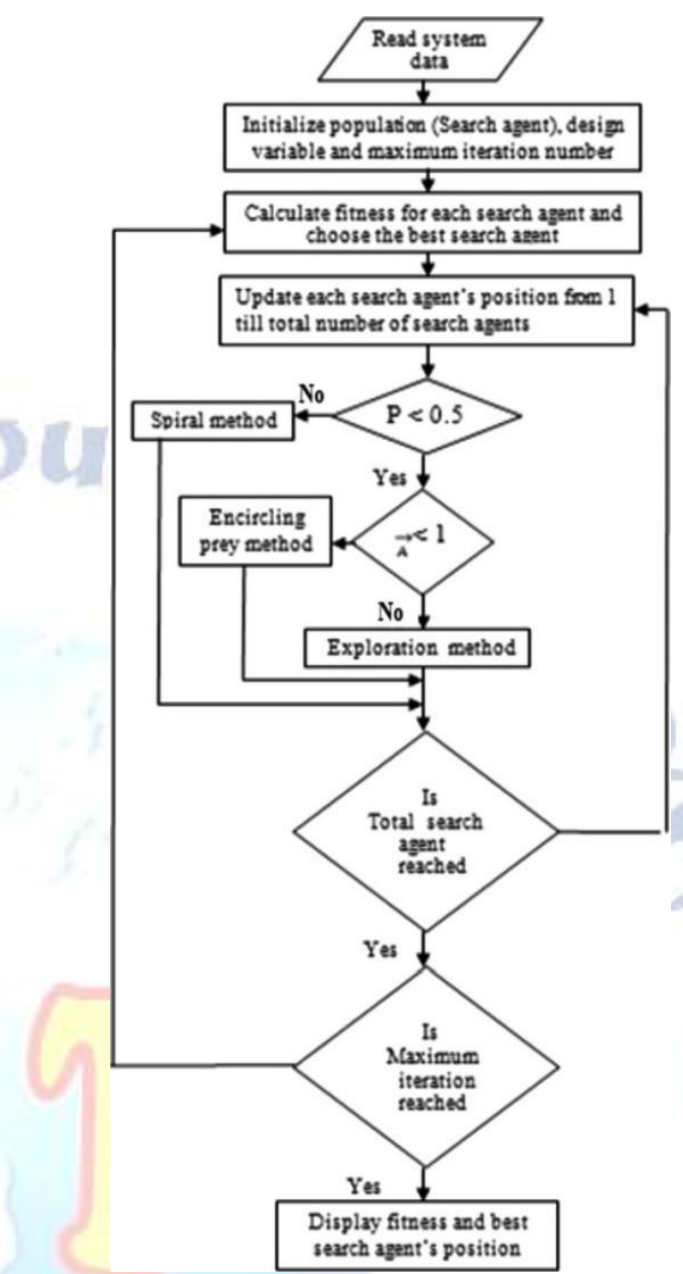

Fig. 4. Flowchart for WOA

\section{RESULT AND DISCUSSIONS}

To control the AVR System is follow up by the suggested controller i.e., PID Controller which are to be enhance for the WOA Algorithm. The terminal voltage feedback profiles $(\Delta \mathrm{Vt})$ retrieved from the AVR system.

\section{Investigation Form on Transient Response}

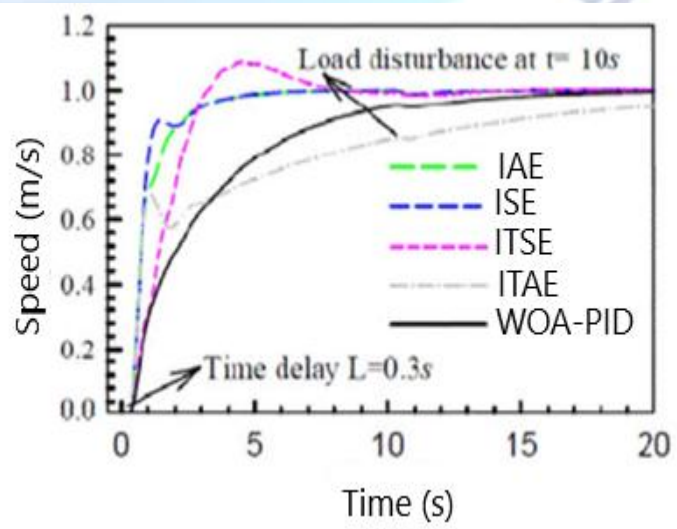

The steady state can be accomplished from the system but before managing that state, the analysis on the transient response, based on the system's performance that can be implemented. To 
reduce the obligation of the representation of the observations based on the damping capability, oscillation and the speed. The observation are yields on the value of $T_{R}$ the speed of the system where the magnitude of $T_{s S}$ and $M_{P}$ opinion the system's nature of oscillations.

Thus, the WOA based on PID controller which entitles the less number of oscillations, improved damping capability (minimum $T_{S S}$ ) and greater reliable (minimum $M_{P}$ ) unit step transient response in sharpness with other optimization techniques are tuned AVR system in PID controller.

\section{Convergence Profile}

The contour of the convergence of the enhanced WOA algorithm is acquire by delineating the minimum FOD value for the PID Controller occupied for the analysis of AVR system against the number of iterations. In Fig. 5, the convergence contour for the enhanced WOA has been characterize by taking into the desired value of $\mathrm{KG}$ $=2.0$ and $\mathrm{W} \mathrm{G}=1.5 \mathrm{~s}$. It might be announced from the figure that the WOA based PID controller has converged in 18 iterations at an FOD value of 0.4548 . Thus, it may be announced from Fig. 5 , the enhanced WOA has delivered promising convergence mobility on tuning of the controller for the inquiry of AVR system.

\section{v. CONCLUSION}

Here in this investigation work, the enhanced WOA tuning PID controller has been expressed used to adjust the terminal voltage of the AVR System to its nominal values under all the proposed loading conditions. To improve the stability of the system the controller is needed in the AVR system and to get fast feedback. For tuning the controller parameters Optimization techniques are used to enhance the performance of AVR system over minimizing the error of the system.From the simulation results and the transient response parameters, it may be reported that the propounded whale optimization technique based on proportional-integral-derivative controller can be employed for these speed control of the system.It is most significant for the definite industrial production of the system. Next, we will study how to improve the performance of the controller and how to apply the controller to the practical engineering techniques. In addition, the implementation of the enhanced WOA algorithm will be tested in detail through other examples.

\section{REFERENCES}

[1] B. Hekimolu, S. Ekinci and S. Kaya, "Optimal PID controller design of DC-DC buck converter using whale optimization algorithm", in Proc.IEEE IDAP, Malatya, Turkey, pp. 1-6, Sep. 2018.

[2] S. Khubalkar, A. Junghare, M. Aware and S. Das, "Modeling and control of a permanent-magnet brushless dc motor drive using a fractional order proportional-integral-derivative controller", Turkish J. Elect. Eng. Comput.Sci., vol. 25, no. 5, pp. 4223-4241, 2017.

[3] I. Podlubny, "Fractional order systems and PID-controllers", IEEE Trans. Automation Control, vol. 44, no. 1, pp. 208-214, Jan. 1999.

[4] S. Das, "Functional Fractional Calculus", 2nd ed. New York, NY, USA: Springer, 2011.

[5] Y. Tenne and C. K. Goh, Computational intelligence in expensive optimization problems, Springer-Verlag Berlin Heidelberg, 2010.

[6] H. Saadat, Power System Analysis, Tata McGraw Hill Series in electrical and computer engineering Ltd, pp. 1-6 New Delhi, 2002.

[7] Shamik Chatterjee, Mohamed Ahmed Dalel and Mahesh Palavalasa, "Design of PID plus second order derivative controller for automatic voltage regulator using whale optimization algorithm" in 2019 3rd International Conference on Recent Developments in Control, Automation \& Power Engineering (RDCAPE)

[8] H. Gozde and M. C. Taplamacioglu, "Comparative performance analysis of artificial bee colony algorithm for automatic voltage regulator (AVR) system", Journal of the Franklin Institute, vol. 348, no. 8, pp. 1927-1946, Oct. 2011.

[9] Z. L. Gaing, "A Particle Swarm Optimization Approach for Optimum Design of PID Controller in AVR System”, IEEE Transactions on Energy Conversion, vol. 19, no.2, pp. 384-391, June 2004.

[10] Ahmed M. Mosaad, Mahmoud A. Attia, Almosataz Y. Abdelaziz, "Whale optimization algorithm to tune PID and PIDA controllers on AVR system" in Ain shams Engineering Journal, Volume 10, Issue 4, December 2019, Pages 755-767

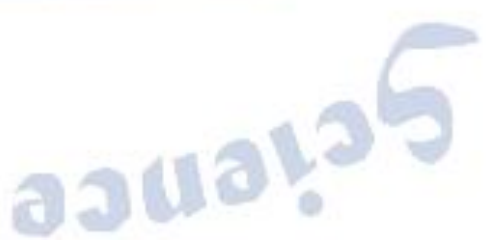

